\title{
Helicoid peripapillary chorioretinal degeneration
}

INSERM

\section{Source}

INSERM. (1999). Orphanet: an online rare disease and orphan drug data base. Helicoid peripapillary chorioretinal degeneration. ORPHA:86813

Helicoid peripapillary chorioretinal degeneration is a rare autosomal dominantly inherited chorioretinal degeneration disease, presenting at birth or infancy, characterized by progressive bilateral retinal and choroidal atrophy, appearing as lesions on the optic nerve and peripheral ocular fundus and leading to central vision loss. Congenital anterior polar cataracts are sometimes associated with this disease. 